\title{
Rasgos Identificativos que promueven la Cultura de la Innovación en las organizaciones
}

\author{
Luz Marina Cifuentes Sánchez ${ }^{1} \&$ Maria Eugenia Londoño Londoño ${ }^{2}$
}

Recibido: 01/09/2017 Aceptado: 26/10/2017

DOI:10.21772/ripo.v36n1a03

\begin{abstract}
Resumen
Si bien existen definiciones sobre cultura organizacional y, por otro lado, de la innovación, es indispensable tratar de entender su conexión como un asunto clave del enfoque de la presente investigación la cual pretende identificar los rasgos de cultura que promueven la cultura de la innovación. Para su elaboración se llevó a cabo un rastreo documental en bases de datos de artículos y libros relacionados con la cultura de la innovación, en los que resultó de interés identificar las opiniones de los autores de los asuntos que promueven la innovación en las organizaciones. Se revisaron 57 textos con fechas de publicación entre los años 1968 y 2016. Como resultado, se agruparon los rasgos que promueven una cultura de la innovación en dieciocho categorías, se utilizó el recuento de los años en que los autores los mencionaron y estos a su vez, se llevaron a un análisis de coocurrencia de palabras y descriptores para identificar la relación directa entre ellos y su nivel de importancia para lograr una cultura con altas características innovadoras.
\end{abstract}

Palabras claves: cultura organizacional, innovación, cultura de la innovación.

\section{Identifying Traits that promote the Innovation Culture in the organizations}

\begin{abstract}
While there are definitions of organizational culture and, on the other hand, of innovation, it's essential to try to understand their connection as a key issue of the present research approach, which aims to identifying the traits that promote the innovation culture. For its elaboration, a documentary tracking was carried out in databases of articles and books related to the innovation culture, in which it was interesting to identify the opinions of the authors of the issues that promote innovation in organizations. We reviewed 57 texts with publication dates between the years 1968 and 2016. As a result, we grouped the features that promote an innovation culture in eighteen categories, we used the count of the years in which the authors mentioned them and these to their Once, they took an analysis of co-occurrence of words and descriptors to identify the direct relationship between them and their level of importance to achieve a culture with high innovative characteristics.
\end{abstract}

Keywords: organizational culture, innovation, innovation culture.

Existe una necesidad continua de desarrollar a los líderes a partir de las expectativas de cambio de la fuerza laboral y los crecientes desafíos que están enfrentando las organizaciones, lo que implica un cambio de paradigmas y de comportamientos al interior de ellas. Esto lleva a replantear aspectos de la cultura organizacional, la conformación de equipos de trabajo $y$, por ende, la forma como las personas conciben los cambios, incorporan nuevos conocimientos y actuaciones y hacen frente a los problemas y situaciones inesperadas en su labor.
Naranjo-Valencia y Calderón-Hernández (2015) y el estudio de tendencias de capital humano realizado por Deloitte en los años 2014 y 2015 a 3.300 líderes organizacionales y de recursos humanos en 106 países (Schatsky \& Schwartz, 2015), indican que los líderes poseen poco conocimiento en la definición de la cultura de sus organizaciones y no se han dado cuenta de cómo desplegarla a lo largo de ella. Frente a los cambios de entorno, la velocidad de satisfacer nuevas demandas para los clientes y la incursión de nuevas tecnologías en el mercado, resulta complejo gestionarla y abordarla.

\footnotetext{
1 Especialista en Gerencia de Proyectos y Magister en Gestión Tecnológica, Profesional Proyectos e Ingeniería. EPM. E-mail: luz.cifuentes@epm.com.co 2 Psicóloga. Especialista en Psicología Organizacional y Salud Ocupacional de la Universidad de Antioquia. Magister en Psicología de las Organizaciones, del Trabajo y de los Recursos Humanos de la Universidad Jaume I de España. Lidera el área de Diseño de Sistemas e Instrumentos Evaluativos en Cincel. E-mail: mariaeugenial@cincel.com.co

Nota: el presente artículo fue publicado en el 2017. Sin embargo, con el fin de mantener la periodicidad de la Revista, el número corresponde al primer semestre del año 2017

Cómo citar este artículo: Cifuentes Sánchez, L. M \& Londoño Londoño, M. E. . (2017). Rasgos Identificativos que promueven la Cultura de la Innovación en las organizaciones. Revista Interamericana de Psicología Ocupacional, 36(1), 36-48, Doi: 10.21772/ripo.v36n1a03
} 
Asimismo, pocas compañías tienen un proceso formal o usan metodologías que les permitan gestionar su cultura e identificar cuáles son rasgos fuertes, débiles o dónde hay inconsistencias (Schatsky \& Schwartz, 2015).

Asimismo, estos autores afirman que la cultura y el compromiso no son solamente temas de las áreas de recursos humanos, sino de negocios. Por ello en el año 2015 lo referente a compromiso y cultura cobraron inmensa validez, hasta convertirse en el desafío número uno en varias organizaciones del mundo. En la época presente es un imperativo para todo líder y todo ejecutivo en la organización (Schatsky \& Schwartz, 2015).

Por otro lado, afirman Naranjo-Valencia y Calderón-Hernández (2015) que este entorno cada vez más competitivo, está invitando a generar nuevas formas de hacer las cosas, en las que se modifica incluso la cultura organizacional. Pues la misma incide en el comportamiento conjunto de la organización, por lo tanto, en sus resultados y, entre ellos, su facultad de innovar y de mantener el mejoramiento continuo. La cultura puede provocar comportamientos innovadores en los miembros de la organización con el propósito de lograr que la gente acepte la innovación como un valor fundamental y también se puede alcanzar un compromiso con ella (Hartmann, 2006).

A pesar del reconocimiento clave de la cultura en la innovación, varios estudios abordan la relación entre los dos conceptos: cultura e innovación, aunque tales acercamientos se han realizado en su mayoría con carácter teórico (Ahmed, 1998; Mumford, 2000; Martins \& Terblanche, 2003; McLean, 2005).

Por otro lado, se han realizado investigaciones previas sobre la cultura en organizaciones de Estados Unidos y Europa y son pocos los estudios que se encuentran sobre la cultura y su integración con la innovación en Latinoamérica. Para el caso de Colombia, la literatura indica que es un país con bajo nivel de innovación organizacional, aunque se han iniciado estudios de cultura de la innovación en la organización Sumicol, del Grupo Corona (NaranjoValencia \& Calderón-Hernández, 2015) como referente para implementar esta práctica en otras organizaciones. Los indicadores de innovación en Colombia, son: el número de solicitudes de patentes en el país no supera las 45 por millón de habitantes, la inversión en innovación sigue siendo inferior a las necesidades del país y solo alcanza $0.23 \%$ del PIB (con proyección del 1\% al año 2018) y la Encuesta de Desarrollo e Innovación Tecnológica (EDIT) del
DANE indica que menos de $1 \%$ de las compañías son estrictamente innovadoras (Competitividad, 2016; 2017).

De esta manera, la presente investigación pretende identificar los rasgos de cultura que promueven la cultura de la innovación en las organizaciones. Para su elaboración se llevó a cabo un rastreo documental en bases de datos de artículos y libros relacionados con la cultura de la innovación, en los que resultó de interés identificar las opiniones de los autores sobre los asuntos que promueven la innovación en las organizaciones. Los rasgos de cultura identificados se agruparon en categorías, se utilizó el recuento de los años en que los autores mencionaron dichos rasgos y estos a su vez, se llevaron a un análisis de coocurrencia de palabras y descriptores para identificar la relación directa entre ellos y, por ende, los que generan mayor impacto en una cultura innovadora.

Es por ello que, como marco principal de la presente investigación, se exponen los conceptos más relevantes que la guían. En la parte inicial se mencionan algunos referentes en relación con la innovación, más tarde se despliega el concepto de cultura organizacional y se finaliza con la noción de cultura de la innovación.

\section{Innovación}

Para el término "innovación" y su relación con la cultura, es necesario determinar su importancia y su integración con la misma. Para algunos autores, como Shumpeter (1942) y el Manual de Oslo (OCDE, 2005), está enfocado hacia la implementación de nuevas cosas, ya sea productos, servicios, mercados, procesos productivos, fuentes de insumo, y cambios en la organización industrial.

De otro lado, otros autores orientan la definición a cosas intangibles como Rothwell (1994), que menciona que es un proceso de aprendizaje o acumulación de know how, y Velasco, Zamanillo y Gurutze (2007) y Madrid, CEIM y Comunidad de Madrid (1995), que consideran la innovación como un intangible que se da a partir de los cambios y necesidades que ha sufrido el mercado hasta el día de hoy.

El término innovación no solo se refiere entonces al concepto de crear cosas nuevas (productos, servicios, procesos de producción, mercados, entre otras posibilidades), sino que es más 
amplio y en él se incluyen asuntos organizacionales como mejorar las formas de trabajo, la aplicación de nuevas metodologías, la disminución de tiempos y actividades que no generan valor en los procesos, productos y servicios y la inyección de tecnología que retribuya un beneficio significativo para las organizaciones.

Existen otras definiciones de autores como Damanpour (1991), Escorsa y Valls (2001) y Ponti (2009), que al referirse a innovaciones las clasifican como radicales, incrementales y menores. Las primeras tienen lugar cuando se incorpora al mercado un producto o servicio que es capaz de generar una clase que no se conocía antes, lo que origina cambios revolucionarios en la tecnología. Las segundas tienen que ver la optimización de procesos y la reducción de costos, y las menores, se enfocan en el logro de pequeñas distinciones en los productos o servicios con respecto a los competidores en un corto plazo. De acuerdo con lo anterior, se identifica que no solo las tareas de innovación de una empresa le corresponden a los departamentos de Innovación y Desarrollo (I+D). Cada una de las personas a partir de su quehacer puede innovar desde las perspectivas de la optimización de procesos, las modificaciones de flujos de trabajo, las nuevas formas de relacionarse y las mejores maneras de hacer las cosas que representen agilidad e impacto en la entrega oportuna del producto o servicio al cliente.

$\mathrm{Y}$, en último lugar, existen definiciones enfocadas hacia las relaciones con el mercado, el cliente, la competencia y la forma como las personas de una organización ponen en práctica nuevas ideas. Las tres primeras se generan a partir de tecnologías existentes o diferenciadoras mediante las que se adquieren amplios conocimientos, se abren nuevas oportunidades de mercado y se intensifican las relaciones con los clientes. A este tipo de innovaciones las denomina Albernathy (1985) arquitectónicas, creadoras de nichos, revolucionarias y rutinarias.

En cuanto a la forma como las personas generan nuevas ideas, Unsworth, Brown y McGuire (2000) proponen una definición de la innovación relacionada con los comportamientos destinados a producir y poner en práctica nuevas ideas, originadas mediante comportamientos proactivos orientados a la acción y al cambio del entorno de cada persona. Sin embargo, dichos autores consideran que el término proactividad es más amplio que la innovación, aunque puede dar lugar a la creatividad y a la innovación, al igual que a otros aspectos como la solución de problemas y el compromiso con las demandas laborales.
Si bien las anteriores definiciones tienen su pertinencia y validez, para efectos de la presente investigación se adopta lo establecido en el Manual de Oslo, pues esta conlleva a un cambio de actuación y de comportamientos en la manera de hacer las cosas:

Introducción de un nuevo o significativamente mejorado, producto (bien y servicio), de un proceso, de un nuevo método de comercialización o de un nuevo método organizativo, en las prácticas internas de la empresa, la organización o el lugar de trabajo (OCDE, 2005, p. 56).

De igual manera, como es fundamental conocer los rasgos de cultura que promueven la innovación, la definición de innovación organizativa cobra relevancia al momento de comprender cuáles son las prácticas, las mejoras en el lugar de trabajo y los métodos organizativos de las relaciones con el cliente y otros grupos de interés (OCDE, 2005) para la conquista de nuevos rumbos.

\section{Cultura organizacional}

Un segundo foco importante y relevante para los efectos de la presente investigación es la definición de cultura organizacional en la que se hizo un recorrido a fin de identificar, a la luz de diferentes autores, la concepción de la misma. Para Schein (1988), la cultura organizacional se define como:

[...] un conjunto de supuestos profundos que no se manifiestan fácilmente, que son creados y desarrollados por un grupo para ayudarles a enfrentar los problemas que se generan en el día a día, ya sea en el ámbito interno como en el externo. (, p. 24)

Desde esta perspectiva, el autor indica que se requiere que el grupo de personas comparta una cantidad significativa de experiencias y tenga una visión compartida del mundo y de su lugar en él.

Si se recurre a Deninson (1991), este autor indica que la cultura de una organización está conformada por valores, creencias, comportamientos, prácticas de gestión y los principios fundamentales del sistema gerencial. Lo anterior va en la vía de lo que nuevamente Schein menciona sobre esta combinación de artefactos que hacen que las personas conciban lo que es apropiado o no (Schein, 1992). Esto a su vez es clave para entender que los comportamientos de las personas hacen que al final la organización sea lo que es. Estos comportamientos se reflejan en la calidad de los productos que ofrece y las experiencias que la organización les hace pasar a sus clientes. 
Esta identidad que se genera es lo que G. Hofstede, G.H. Hofstede y Minkov (1999) definen como rasgos culturales o programación mental colectiva que distingue a los trabajadores y a la organización como tal.

\section{Cultura de la innovación}

Si bien se ha hablado de cultura y, por otro lado, de la innovación, es clave tratar de entender su conexión. De acuerdo con Naranjo-Valencia y Calderón-Hernández (2015), diversas perspectivas teóricas, como la teoría de recursos y capacidades organizacionales, sustentan el vínculo.

Barlett y Ghoshal (1987) también presentan su punto de vista basado en la interrelación cultural que vincula las culturas de origen de las personas con las de adopción, y afirman que este mestizaje constituye una fuente de conocimiento e innovación que, al sumarle lo que mencionan Amabile (1997) y Gurteen (1998), en dicho mestizaje se debe favorecer un ambiente de trabajo que estimula la comunicación y los flujos de información en la organización y de la organización con el entorno. En este sentido es válido determinar que para lograr una cultura con las mencionadas características se requiere contar con un talento humano creativo que, visto desde la perspectiva del colectivo, se considera un comportamiento innovador, por lo que podría entenderse en la cultura organizacional como un rasgo, una característica, una marca o, incluso, un proceso que la hace particular.

Por su parte, Cameron y Quinn (1999) plantean que la cultura, como fuente de innovación, se caracteriza por sus niveles de adaptabilidad, flexibilidad, creatividad, convivencia con la incertidumbre y ambigüedad de la información, ausencia de poder centralizado, énfasis en la individualidad, riesgo y anticipación a los cambios o transformaciones organizacionales. De igual manera, extienden el concepto de cultura organizacional a un modelo de valores en competencia (MVC) que especifica cuatro tipos de cultura en dos dimensiones: estabilidad versus flexibilidad y valores contrapuestos. Los tipos de cultura que presentan dichos autores son:

Cultura de clan: enfatiza en el desarrollo humano, el trabajo en equipo y la participación de los trabajadores.

Cultura adhocrática: valora en lo fundamental la iniciativa, la creatividad y la asunción de riesgos.
Cultura racional: también llamada de mercado. Potencia la consecución de los objetivos ambiciosos.

Cultura jerárquica: los valores más importantes son la eficiencia, el cumplimiento de las normas y la formalización de los procesos.

Al tener en cuenta diferentes mediciones realizadas por Naranjo-Valencia y CalderónHernández (2015), los resultados muestran que los tipos de cultura mencionados por Cameron y Quinn tienen efectos distintos sobre la innovación; la cultura de tipo adhocrático favorece la innovación mientras que la de tipo jerárquico la afecta en sentido negativo.

Es así como, a partir de dicho planteamiento, la cultura se ha identificado como un habilitador de la innovación que se puede favorecer o entorpecer según rasgos culturales que existan en una organización, los cuales se entenderán en la presente investigación como:

Las características de la cultura organizacional tales como: percepciones, valores, creencias, actitudes, hábitos, tradiciones que son esenciales para la interacción dentro y entre los grupos existentes en las organizaciones que fundamentan el sistema gerencial (Deninson, 1991; Schein 1992).

Por ello se hace importante identificar los rasgos culturales que la promueven y reconocer su importancia a través de las prácticas que las organizaciones tienen establecidas como la mejor forma de intervenirla (Hofstede et al., 1999).

\section{Metodología}

La presente investigación fue de carácter exploratorio y descriptivo y se dividió en dos fases:

La fase inicial permitió identificar cuáles son los rasgos de cultura y los factores de equipos de trabajo e individuales que promueven la cultura de la innovación a partir de un rastreo documental en bases de datos de artículos y libros relacionados con la cultura de la innovación, como los estudios realizados por Naranjo y sus coautores $(2011 ; 2015)$, López González (2014), Robledo Velásquez y sus coautores (2013; 2016), Cincel (2014; 2015), Unsworth y sus coautores $(2001 ; 2003 ; 2005 ; 2010)$ y Schatsky y Schwartz (2015). En ellos resultó de interés identificar 
las opiniones de los autores acerca de los asuntos que promueven la innovación en las organizaciones. Se revisaron 57 textos con fechas de publicación entre los años 1968 y 2016.

En el proceso de recolección de información se tuvieron en cuenta palabras claves como: cultura organizacional, innovación y cultura de la innovación. Asimismo, fue relevante analizar los factores de los equipos de trabajo e individuales que promueven la innovación según el modelo conceptual desde el punto de vista psicosocial desarrollado por Cincel (2014; 2015) y López González (2014), el cual fue construido a partir de los planteamientos de la teoría y la investigación multinivel. Los factores de equipo analizados para esta investigación fueron: cohesión, potencia, liderazgo y clima de aprendizaje de equipo; y los factores individuales analizados fueron: autoeficacia general, autoeficacia creativa, desarrollo psicológico, clima de aprendizaje psicológico y compromiso (Engagement).

Los rasgos encontrados se agruparon en 22 categorías, diferenciándolos enrasgosorganizacionales y rasgos internos que son evidenciables en los equipos de trabajo, según lo indicado en el modelo multinivel. El nombramiento de cada una de las categorías definidas, fueron acompañadas por el asesoramiento de un experto.

Para dar mayor fuerza al análisis se utilizó el recuento de los años en que los autores mencionaron los rasgos de cultura que promueven una cultura de la innovación encontrados en la literatura. Dichos rasgos se llevaron a una tabla en el que se describió: el rasgo encontrado en la literatura, los autores que lo mencionaban, el número de menciones encontradas y la categoría asignada, por ejemplo: Orientación a la Innovación, Innovación abierta, Trabajo en equipo, entre otros (Ver Tabla 1).

Cada categoría se llevó a un análisis de coocurrencia de palabras. Esta metodología permite saber cuándo dos palabras van juntas en el título, en el resumen o en el número de descriptores o de identificadores; si aparecen juntas significa que existe una relación directa entre ellas. Para la presente investigación se analizó la proximidad entre los descriptores e identificadores de los rasgos, es decir, si se encontraba en el texto un rasgo que se relacionaba con otro en su definición, se resaltaba la relación directa por medio de una flecha. Si la coocurrencia es baja quiere decir que no hay relación sino distancia (Escorsa, 2008) (Ver figura 1).
De acuerdo con la proximidad que tenían los rasgos se representaron en forma gráfica en un plano circular de la siguiente manera: la categoría (detallada por el nombre y por medio de un círculo o nodo), el número de menciones de cada categoría (identificada por un paréntesis y un número que se encuentra después del nombre de la categoría) y las relaciones entre las categorías (establecidas por medio de unas flechas que conectan ambos sentidos entre una categoría y otra) (Ver figura 1). Lo anterior, permitió evidenciar dónde se concentra la importancia que le dieron los autores a los rasgos. Esta representación gráfica recibe el nombre de mapa tecnológico (Escorsa, 2008).

En la segunda y última fase, de carácter analítico, se analizaron las categorías según el número de menciones encontradas. Este análisis se hizo de mayor a menor, indicando que los rasgos que tuvieron mas de 10 puntos, son referentes para lograr una cultura innovadora. De igual forma, se analizaron las relaciones entre las categorías para determinar si una de ellas se trabaja, está favoreciendo otra.

Los resultados obtenidos se documentaron y a partir del panorama encontrado, se plantearon una serie de recomendaciones con el fin de promover la cultura de la innovación en una organización de cualquier sector.

\section{Resultados}

Tras el análisis descriptivo de los rasgos de cultura que promueven la innovación en las organizaciones, se identificaron 18 que para los autores consultados presentan un mayor número de repeticiones en la literatura. A continuación, se describen los rasgos de cultura que promueven la innovación clasificados en dieciocho categorías, según el número de repeticiones identificadas por los autores consultados (Tabla 1). 
Tabla 1

\begin{tabular}{|c|c|c|c|}
\hline $\begin{array}{c}\text { Rasgos de cultura innovadora en las } \\
\text { organizaciones }\end{array}$ & Autor(es) & No. & Categoría \\
\hline $\begin{array}{l}\text { Objetivos desafiantes, operaciones y tareas } \\
\text { asignadas son retos para superar, compromiso en } \\
\text { el cumplimiento de las metas }\end{array}$ & Cotec (2005) & \multirow[t]{7}{*}{21} & \multirow[t]{7}{*}{$\begin{array}{l}\text { Orientación a la innovación: existencia espacios } \\
\text { que impulsen la creatividad y planes y programas que } \\
\text { favorecen la innovación }\end{array}$} \\
\hline Obsesión por el producto & Barba (2011) & & \\
\hline $\begin{array}{l}\text { Orientación al logro, flexibilidad mental y } \\
\text { capacidad de asombro }\end{array}$ & $\begin{array}{c}\text { Naranjo-Valencia y Calderón- } \\
\text { Hernández (2015) }\end{array}$ & & \\
\hline Libertad y autonomía & $\begin{array}{c}\text { Shrivastava y Souder (1987), Ahmed } \\
\text { (1998), Arad, Hanson y Schneider } \\
\text { (1997), Martins y Terblanche (2003), } \\
\text { McLean (2005), Jamrog, Vickers y } \\
\text { Beer (2006) }\end{array}$ & & \\
\hline $\begin{array}{l}\text { Creatividad, iniciativa y espíritu emprendedor } \\
\text { (emprendimiento) }\end{array}$ & $\begin{array}{l}\text { Wallach (1983); Shrivastava y Souder } \\
\text { (1987); Claver, Llopis, Garcia y } \\
\text { Molina (1998); Schneider, Gunnarson } \\
\text { y Niles-Jolly (1994), Canalejo (1995), } \\
\text { Martins y Terblanche (2003), McLean } \\
\text { (2005), Jamrog et al. (2006); Cameron } \\
\text { y Quinn (1999), Naranjo-Valencia y } \\
\text { Calderón-Hernández (2015) }\end{array}$ & & \\
\hline $\begin{array}{l}\text { Ambiente de trabajo que impulse la creatividad } \\
\text { como actitud favorable a la innovación }\end{array}$ & Twiss (1986) & & \\
\hline $\begin{array}{l}\text { Percepción del trabajo como hecho divertido, } \\
\text { estimulante y emocionante e incorporación de } \\
\text { sistemas de pensamiento creativo }\end{array}$ & Ponti (2009) & & \\
\hline Apertura a la toma de riesgos & $\begin{array}{l}\text { Aiman-Smith, Goodrich, Roberts y } \\
\text { Scinta (2005) }\end{array}$ & \multirow[t]{6}{*}{12} & \multirow{6}{*}{$\begin{array}{l}\text { Asunción del riesgo: existencia en la organización } \\
\text { de una metodología de riesgos que analice en forma } \\
\text { periódica la estrategia y la incertidumbre que se derivar } \\
\text { de la actividad innovadora }\end{array}$} \\
\hline $\begin{array}{l}\text { Estrategia organizacional: toma de riesgos, } \\
\text { compromiso y enfoque }\end{array}$ & Saleh y Wang (1993) & & \\
\hline Aceptación del riesgo & $\begin{array}{l}\text { Naranjo-Valencia y Calderón- } \\
\text { Hernández (2015), }\end{array}$ & & \\
\hline & López González et al., (2014) & & \\
\hline Asunción de riesgo & $\begin{array}{l}\text { Wallach (1983), Claver et al. (1998), } \\
\text { Martins y Terblanche (2003), McLean } \\
\text { (2005), Jamrog et al. (2006), Cameron } \\
\text { y Quinn (1999) }\end{array}$ & & \\
\hline $\begin{array}{l}\text { Búsqueda del riesgo, tolerancia al mismo y } \\
\text { tolerancia al fracaso }\end{array}$ & Barba (2011), Bueno y Morcillo (2003) & & \\
\hline $\begin{array}{l}\text { Estructura organizacional que soporta las } \\
\text { comunicaciones }\end{array}$ & $\begin{array}{l}\text { Aiman-Smith, Goodrich, Roberts y } \\
\text { Scinta (2005) }\end{array}$ & \multirow[t]{7}{*}{12} & \multirow{7}{*}{$\begin{array}{l}\text { Comunicación y participación: existencia en la } \\
\text { organización de una estructura organizacional que } \\
\text { soporta las comunicaciones que les permitan a los } \\
\text { trabajadores expresar opiniones, proponer nuevas ideas } \\
\text { y tomar decisiones en un ambiente libre, de confianza } \\
\text { y de apertura, como medio para desarrollar nuevos } \\
\text { conocimientos y destrezas adicionales }\end{array}$} \\
\hline Capacidad de confrontación & $\begin{array}{c}\text { Naranjo-Valencia y Calderón- } \\
\text { Hernández (2015) }\end{array}$ & & \\
\hline $\begin{array}{l}\text { Confianza en proponer ideas y opiniones, permitir } \\
\text { el debate en ambiente libre y de apertura y } \\
\text { comunicación interna }\end{array}$ & Cotec (2005) & & \\
\hline $\begin{array}{l}\text { Estructura organizacional que facilita los canales } \\
\text { de comunicación }\end{array}$ & Twiss (1986) & & \\
\hline Herramientas de comunicación interna & Ponti (2009) & & \\
\hline $\begin{array}{l}\text { Ambiente de trabajo que estimule la comunicación } \\
\text { y los flujos de información al interior de la } \\
\text { organización y con el entorno }\end{array}$ & Amabile (1997), Gurteen (1998) & & \\
\hline $\begin{array}{l}\text { Participación: habilitación, orientación al equipo y } \\
\text { desarrollo de destrezas }\end{array}$ & $\begin{array}{c}\text { Factor (2010), Naranjo-Valencia y } \\
\text { Calderón-Hernández (2015), Cameron } \\
\text { y Quinn (1999), Claver et al. (1998), } \\
\text { McLean (2005) }\end{array}$ & & \\
\hline
\end{tabular}


Rasgos de cultura innovadora en las organizaciones

Morcillo Ortega (2007), Jakobiak

(1992), Rouach (1996), Lesca (1994),

Martinet y Marti (1995), Detert, Scroeder y Muriel (2000)

estrategia, generación de conocimiento y obtención de innovaciones

Inteligencia de negocios

Aiman-Smith, Goodrich, Roberts y Scinta (2005)

Toma de decisiones basadas en el comportamiento Martins y Terblanche (2003), McLean del mercado y en nuevas tendencias

(2005), Cotec (2005)
No.

10 Vigilancia tecnológica e inteligencia competitiva: proceso organizado, selectivo y permanente de captar información del exterior y de la propia organización sobre ciencia y tecnología, seleccionarla, analizarla, difundirla y comunicarla, para convertirla en conocimiento para tomar decisiones con menor riesgo y poder anticiparse a los cambios

\section{Compañerismo y apertura}

Trabajo en equipo
Saleh y Wang (1993)

Canalejo (1995), Arad et al. (1997),

Martins y Terblanche (2003), McLean

(2005); Jamrog et al. (2006), Cameron y Quinn (1999)

Colaboración abierta

Respeto, capacidad y confianza

Estructura organizacional flexible que promueva el ambiente colaborativo

Flexibilidad Barba (2011)

Naranjo-Valencia y CalderónHernández (2015)

Saleh y Wang (1993), Bueno y Morcillo (2003)

Cameron y Quinn (1999), Arad et al. (1997), Martins y Terblanche (2003)

Ausencia de poder centralizado y de cadenas de Cameron y Quinn (1999)

mando establecidas

\begin{tabular}{l}
\hline Centros de poder flexibles \\
\hline Estructura poco jerárquica \\
\hline Inteligencia colectiva externa en beneficio de la \\
innovación, orientada al exterior \\
\hline Orientación al mercado
\end{tabular}

Cotec (2005)

Ponti (2009)

Ponti (2009); Kimberly y Evanisko (1981)

Naranjo-Valencia y CalderónHernández (2015)

Orientación estratégica hacia el cliente

Canalejo (1995), Martins y Terblanche

(2003), Jamrog et al. (2006)

\begin{tabular}{ll}
\hline Abierta al mundo y a la variedad de organización & Barba (2011) \\
\hline Contacto con clientes y otros grupos de interés & Ponti (2009) \\
\hline $\begin{array}{l}\text { Adaptabilidad: creación de cambios, enfoque en el } \\
\text { cliente y aprendizaje organizaciónrial }\end{array}$ & Factor (2010)
\end{tabular}

cliente y aprendizaje organizaciónrial

\begin{tabular}{lc}
\hline Adaptabilidad & Cameron y Quinn (1999) \\
\hline Tolerancia a la incertidumbre & Cotec (2005) \\
\hline Entusiasmo por el cambio & Barba (2011) \\
\hline Convivencia con la incertidumbre y la ambigüedad & Cameron y Quinn (1999)
\end{tabular}

de la información

\begin{tabular}{lc}
\hline Fomento de la disposición al cambio & Bueno y Morcillo (2003) \\
\hline Apoyo a las ideas e iniciativas de cambio & Cotec (2005) \\
\hline Suficiencia de recursos & $\begin{array}{c}\text { Ahmed (1998); McLean (2005); } \\
\text { Jamrog et al. (2006) }\end{array}$ \\
\hline Apoyo a la generación de nuevas ideas & Bueno y Morcillo (2003) \\
\hline Aprendizaje continuo & $\begin{array}{c}\text { Lemon y Sahota (2004), Canalejo } \\
\text { (1995), Martins y Terblanche (2003), } \\
\text { Bueno y Morcillo (2003) }\end{array}$ \\
\hline
\end{tabular}

Aprendizaje organizativo que impulsa el trabajo en equipo, la interdisciplinariedad, la integridad cultural, las estructuras organizativas colaborativas y las iniciativas de cooperación externa

Desarrollo humano y concepto del ser humano

Libertad en que los miembros de la organización trabajen en áreas de su interés

Morcillo Ortega (2007)

Cameron y Quinn (1999)

Cotec (2005); Child (1990)

Dirección y gestión de los recursos humanos,

dirección y gestión por competencias, inteligencia

cultural, contrato social, motivación, creatividad

Morcillo Ortega (2007)
8 Trabajo en equipo: existencia de mecanismos de integración y relacionamiento entre los trabajadores que faciliten el compañerismo, la apertura, la colaboración, el respeto y la confianza en la organización

8 Estructura organizacional flexible: existencia en la organización de centros de poder flexibles que promuevan el ambiente colaborativo (ausencia de poder centralizado y grandes cadenas de mando)

8 Innovación abierta: capacidad para crear y compartir conocimiento con el cliente y profesionales externos para el desarrollo de nuevas soluciones. Asimismo, poner a disposición del mercado canales internos y externos para ofrecer productos y tecnologías innovadoras que contribuyan al logro de la estrategia organizacional

6 Adaptación a los cambios: percepción de la velocidad con la que la organización asume los cambios propuestos por los clientes y el entorno y la forma como convive con la incertidumbre

5 Asignación de recursos: recursos estimados para la actividad innovadora que permita materializar ideas e iniciativas de cambio

5 Aprendizaje continuo: grado en el que se estimula el aprendizaje individual y colectivo a través de la cooperación y la comunicación de nuevos conocimientos e ideas en los equipos de trabajo
4 Contribución al desarrollo del ser humano: percepción de que la organización tiene como centro el ser humano de tal modo que trabajar en sus áreas de interés, gestiona sus competencias, la inteligencia cultural, el contrato social, la motivación y la creatividad 


\begin{tabular}{|c|c|c|c|}
\hline $\begin{array}{c}\text { Rasgos de cultura innovadora en las } \\
\text { organizaciones }\end{array}$ & Autor(es) & No. & Categoría \\
\hline Liderazgo & $\begin{array}{l}\text { Naranjo-Valencia y Calderón- } \\
\text { Hernández (2015), Cotec (2005), Twiss } \\
\text { (1986), Ponti (2009) }\end{array}$ & 4 & $\begin{array}{l}\text { Estilo de liderazgo: grado en que la organización } \\
\text { fomenta un liderazgo que acompaña a sus trabajadores, } \\
\text { conoce las oportunidades de mejora, favorece una } \\
\text { visión clara de futuro y prevé las consecuencias de las } \\
\text { actuaciones propias y del equipo }\end{array}$ \\
\hline Ilusión compartida & Ponti (2009) & \multirow[t]{4}{*}{4} & \multirow{4}{*}{$\begin{array}{l}\text { Propósitos y significados comunes: contar con una } \\
\text { visión compartida que les permita a los trabajadores tener } \\
\text { una comprensión unificada de la intención organizacional } \\
\text { y su dirección estratégica (misión, visión, valores, metas } \\
\text { y objetivos) }\end{array}$} \\
\hline $\begin{array}{l}\text { Misión, intención y dirección estratégica, metas, } \\
\text { objetivos y visión }\end{array}$ & Factor (2010) & & \\
\hline Planeación estratégica & $\begin{array}{l}\text { Aiman-Smith, Goodrich, Roberts y } \\
\text { Scinta (2005) }\end{array}$ & & \\
\hline $\begin{array}{l}\text { Estilo corporativo: definición del proyecto } \\
\text { organizacional, asunción de riesgos, capital } \\
\text { relacional, estilo de dirección participativo }\end{array}$ & Morcillo Ortega (2007) & & \\
\hline Sistema de recompensas & Saleh y Wang (1993) & \multirow[t]{3}{*}{3} & \multirow{3}{*}{$\begin{array}{l}\text { Recompensas: reconocimiento al buen trabajo como } \\
\text { mecanismo de regulación social, salarial y emocional y } \\
\text { exigencia y ascenso por méritos }\end{array}$} \\
\hline Reconocimiento y exigencia de la labor realizada & $\begin{array}{l}\text { Naranjo-Valencia y Calderón- } \\
\text { Hernández (2015) }\end{array}$ & & \\
\hline Ascenso por méritos & Barba (2011) & & \\
\hline Valores, creatividad e innovación & Martins y Terblanche (2003) & \multirow[t]{3}{*}{3} & \multirow{3}{*}{$\begin{array}{l}\text { Valores compartidos: cualidades o virtudes atribuidas } \\
\text { a una persona que influyen en que sea apreciada } \\
\text { y considerada; ejemplo: creatividad, innovación, } \\
\text { consistencia, ética y responsabilidad }\end{array}$} \\
\hline $\begin{array}{l}\text { Consistencia en la incorporación de valores básicos, } \\
\text { acuerdos y coordinación e integración de acciones } \\
\text { que fomenten los valores organizacionales }\end{array}$ & Factor (2010) & & \\
\hline Ética y responsabilidad social & $\begin{array}{l}\text { Naranjo-Valencia y Calderón- } \\
\text { Hernández (2015) }\end{array}$ & & \\
\hline Aceptación de la diversidad & $\begin{array}{l}\text { Naranjo-Valencia y Calderón- } \\
\text { Hernández (2015) }\end{array}$ & \multirow[t]{2}{*}{2} & \multirow{2}{*}{$\begin{array}{l}\text { Multidisciplinariedad: diversidad de conocimientos } \\
\text { y culturas que aporten a la heterogeneidad de una } \\
\text { organización para favorecer el aprendizaje continuo y la } \\
\text { innovación abierta }\end{array}$} \\
\hline Interrelación cultural & Barlett y Ghoshal (1987) & & \\
\hline Mesura en la sobrecarga de trabajo & Cotec (2005) & 1 & $\begin{array}{l}\text { Fomento del equilibrio entre la vida personal y la } \\
\text { laboral: promoción de una cultura que valore la vida } \\
\text { privada y el trabajo realizado en una organización }\end{array}$ \\
\hline Clima organizacional favorable a la colaboración & Twiss (1986) & 1 & $\begin{array}{l}\text { Clima organizacional favorable: percepciones } \\
\text { colectivas que construyen los miembros de un equipo } \\
\text { o de una organización en relación con las distintas } \\
\text { realidades de su trabajo }\end{array}$ \\
\hline
\end{tabular}

Nota. No.=Número de repeticiones.

Un hallazgo importante fue el número de menciones que dan los autores a los rasgos de cultura que promueven la innovación: orientación a la innovación, asunción del riesgo, vigilancia tecnológica, innovación abierta y adaptación a los cambios propuestos por sus grupos de interés. Los rasgos estructura organizacional flexible y la asignación de recursos, se evidencian como elementos organizacionales claves para facilitar la generación de innovaciones. Le siguen en su orden de importancia, con seis repeticiones: la adaptación a los cambios; con cinco repeticiones: asignación de recursos y aprendizaje continuo; con cuatro repeticiones: contribución al desarrollo del ser humano, estilo de liderazgo y propósitos y significados comunes en los equipos de trabajo; con tres repeticiones: recompensas y valores compartidos; con dos repeticiones: multidisciplinariedad; y finalmente con una mención: fomento del equilibrio entre la vida personal y la laboral y clima organizacional favorable. Todos estos organizados de mayor a menor, según lo indicado en la tabla 1.

Asimismo, en el análisis de coocurrencia de palabras y descriptores, se muestra la relación directa entre las variables internas de la organización, las cuales tienen que ver con aspectos en los que las personas comprenden su contribución a la innovación y con aquellos que contribuyen al desarrollo del ser y del hacer. Para el caso de la comprensión del termino de innovación en las organizaciones, se evidencia la importancia de generar espacios que estimulen el trabajo en equipo, la comunicación y la participación de los trabajadores en el desarrollo de nuevas ideas y el aprendizaje continuo mediante la diversidad de conocimientos y culturas, de modo tal que los propósitos, significados y valores sean compartidos entre los trabajadores. Para el caso del desarrollo del talento humano, una organización con una cultura que promueva la innovación se preocupa por un estilo de liderazgo que acompañe a los trabajadores, conozca sus oportunidades de mejora, favorezca una visión clara de futuro y prevea las consecuencias propias y del equipo, lo mismo que por el reconocimiento al buen trabajo como mecanismo de regulación social, según lo indican Naranjo-Valencia y CalderónHernández (2015), Cotec (2005), Twiss (1986) y 


\section{Ponti (2009).}

Si bien los rasgos culturales relacionados con el fomento del equilibrio entre la vida personal y laboral y el clima organizacional favorable presentan una repetición, existen relaciones directas con aquellos rasgos que tienen mayores menciones dadas por autores tales como: trabajo en equipo, comunicación, participación y contribución al desarrollo del ser humano. Lo anterior permite identificar estos rasgos como resultantes de los demás rasgos desarrollados, pues finalmente será la percepción de los trabajadores la que precisa si la organización valora el trabajo realizado y por ende si la innovación en su hacer si se está desplegando.

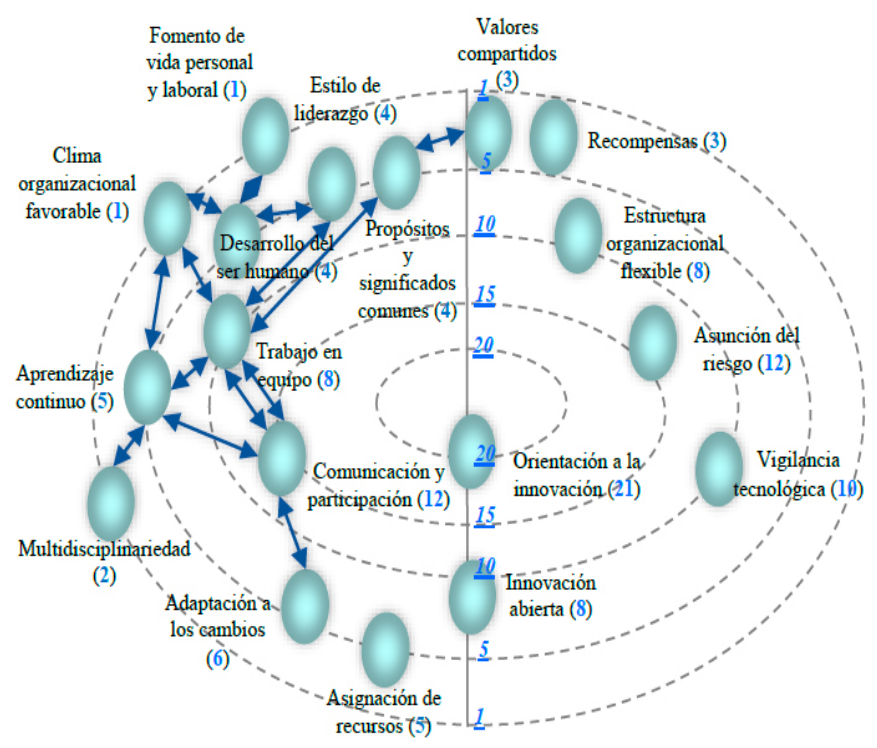

Figura 1: Relaciones entre rasgos de cultura que promueven la innovación. Fuente: elaboración propia

\section{Discusión}

Los rasgos identificativos de cultura que promueven la innovación indicados por los autores están relacionados con orientación a la innovación, la asunción del riesgo, la vigilancia tecnológica, la innovación abierta, la adaptación a los cambios y la estructura organizacional flexible. Para que estos se den es preciso que en las organizaciones los trabajadores perciban que prevalecen espacios para la innovación, que existe un proceso organizado de captar información del interior y del exterior de ella en términos de los avances de ciencia y tecnología, y que los trabajadores en sus actividades laborales, pueden encontrar oportunidades para proponer nuevos asuntos que impacten los productos o servicios que ofrecen (EPM, Gerencia Cultura Organizacional, 2015).
Lo anterior coincide con lo que indican Kimberly y Evanisko (1981) y Ponti (2009), que plantean que las organizaciones orientadas al exterior suelen contar con una variedad de mecanismos de obtención de información del entorno organizacional y la inteligencia colectiva externa en beneficio de la innovación. Este argumento es compartido por Detert et al. (2000), que dice que las organizaciones innovadoras buscan de forma activa nuevas ideas en fuentes de información distintas a las tradicionales. Para dichos autores, las organizaciones que se centran en aspectos externos como los clientes, los competidores y el entorno en general, la innovación se basa en la información obtenida de las fuentes externas mencionadas y en las buenas prácticas seguidas por el resto de las organizaciones en espacios que impulsen la creatividad y los planes y programas en pro de la innovación.

Contar con cultura de la innovación en una organización, propende por la ausencia de poder centralizado y de cadenas de mando establecidas (Cameron \& Quinn, 1999), centros de poder flexibles (Cotec, 2015) y poca jerarquía (Ponti, 2009). Esto contribuyea que se susciteporunambientecolaborativo y asociativo entre los líderes y trabajadores (Saleh \& Wang, 1993; Bueno \& Morcillo, 2003).

El rasgo de innovación abierta, invita a las organización a tener una orientación al mercado (Naranjo-Valencia \& Calderón-Hernández, 2015) y al cliente como se ha indicado (Canalejo, 1995). Este rasgo estimula a la organización para que tenga contacto permanente con los grupos de interés.

Otro aspecto favorable para la promoción de una cultura innovadora, es el optimismo por la aceptación de los cambios por parte de los trabajadores y la mentalidad de riesgo. Lo anterior se asocia con la tolerancia a la incertidumbre y entusiasmo por lo nuevo como lo propone el estudio Tecnología e Innovación en España realizado en el año 2015 (Cotec, 2015).

En cuanto a los rasgos de cultura que promueven la innovación interna como el trabajo en equipo, la comunicación y la participación de los trabajadores en el desarrollo de nuevas ideas y el aprendizaje continuo, se encontró como aspecto favorable la facilidad que debe tener un equipo para trabajar juntos, tener capacidad de confrontación (NaranjoValencia \& Calderón-Hernández, 2015), confianza en proponer nuevas ideas y opiniones, tener debates en ambiente libre y de apertura, comunicación asertiva (Cotec, 2005), orientación al equipo y desarrollo de 
destrezas compartidas (Naranjo-Valencia \& Calderón-Hernández, 2015; Cameron \& Quinn, 1999; Claver et al., 1998; McLean, 2005).

Varios autores destacan la flexibilidad como uno de los valores que más se asocia a las culturas innovadoras y que los factores asociados con la creatividad, la autonomía o la asunción de riesgos pueden resultar difíciles de promover cuando la organización pone su énfasis en la normatividad, la estabilidad y el control (Arad et al., 1997; Cameron y Quinn, 1999; Martins \& Terblanche, 2003).

En cuanto al aprendizaje continuo, este rasgo ayuda a impulsar el trabajo en equipo, la interdisciplinariedad y la interdependencia (Morcillo, 2007). Frente a este rasgo, es preciso tener en cuenta los beneficios que se obtienen al delegar la autoridad y la participación de los trabajadores en la toma de decisiones para fomentar el aprendizaje y el desarrollo profesional de los mismos. Lo anterior los hace más capaces de asumir los riesgos asociados con la innovación (Child, 1990), lo cual va en la vía de tener una marca de liderazgo que reconozca con mayor frecuencia a los empleados por la labor realizada y fomente en los equipos capacidades requeridas por el ADN innovador (Naranjo-Valencia \& CalderónHernández, 2015).

\section{Conclusiones}

El análisis de la bibliografía encontrada permitió identificar los aspectos que más se repitieron como aquellos que los autores reconocen como antecedentes de la innovación y, en investigaciones como las de Cincel (2014; 2015) y López (2015), como los que tienen mayor impacto sobre el proceso innovador.

Asimismo, la proximidad de los rasgos graficados en el mapa tecnológico de la figura 1, permitió hacer un análisis más sencillo de los resultados encontrados en la literatura. En ellos se puede observar la mayor concentración de importancia que les dan los autores a aquellos asuntos que promueven la innovación. A su vez, se pueden observar con claridad las relaciones entre ellos.

A partir de los resultados encontrados en la identificación de los rasgos de cultura que promueven la innovación se puede concluir que, los rasgos que promueven la cultura de la innovación en una organización con mayor impacto son en su orden: orientación a la innovación, asunción al riesgo, comunicación y la participación de los trabajadores, vigilancia tecnológica e inteligencia competitiva, trabajo en equipo, estructura organizacional flexible y la innovación abierta.

Para futuros estudios, se propone llevar a cabo entrevistas a profundidad a líderes que trabajen en empresas de carácter innovador, pues resulta interesante probar las relaciones de sus percepciones con los resultados suministrados por los instrumentos utilizados, ya sea para afianzar asuntos conocidos o comprender asuntos nuevos positivos que los ayuden a concretar, intensificar y potencializar en el desarrollo de innovaciones.

\section{Referencias}

Aiman-Smith, L., Goodrich, N., Roberts, D., \& Scinta, J. (2005). Assessing your organization's potential for value innovation. Research Technology Management, 2(6), 37-42.

Ahmed, P. (1998). Culture and climate for innovation. European Journal of Innovation Management, 1(1), 30-3. Doi: $10.1108 / 14601069810199131$

Albernathy, W. J. (1985). Innovation: mapping the winds of creative destruction. Research Policy, 14(1), 3-22. Doi: 10.1016/00487333(85)90021-6

Amabile, T. M. (1997). Motivanting creativity in organizations: on doing what you love and loving what you do. California Management Review, 40(1), 39-58.

Arad, S., Hanson, M. A., \& Schneider, R. (1997). A framework for the study of relationships between organizational characteristics and organizational innovation. The Journal of Creative Behavior, 31(1), 42-58. doi: 10.1002/ j.2162-6057.1997.tb00780.x

Barba, E. (2011). Innovación: 100 Consejos para inspirarla y gestionarla. Barcelona: Libros de cabecera.

Barlett, C. A., \& Ghoshal, S. (1987). Managing across borders: new strategy requirements. Sloan Management Review, 28(4), 7-17.

Bueno, E., \& Morcillo, P. (2003), "Cultura e innovación: conexión perfecta”, Madri $+\mathrm{d}$, num. 15, febrero-marzo (edición electrónica).

Cameron, K., \& Quinn, R. (1999). Diagnosing and changing organizational culture based on the competing values framework. Reading, MA: Addison-Wesley. 
Canalejo, M. (1995). Innovación organizativa en Alcatel Standard Eléctrica S. A. Madrid: V Congreso Nacional de ACEDE (Asociación Científica de Economía y Dirección de Empresa).

Centro de Investigación en Comportamiento Organizacional, Cincel S. A. S. (2014). Estudio diagnóstico del clima organizacional $y$ del compromiso laboral. Medellín: Cincel.

Centro de Investigación en Comportamiento Organizacional, Cincel S. A. S. (2015). Estudio diagnóstico del clima organizacional $y$ del compromiso laboral. Medellín: Cincel.

Claver, E., Llopis, J., Garcia, D., \& Molina, H. (1998). Organizational culture for innovation and new technological behavior. The Journal of High Technology Management Research, 9(1), 5568.

Child, J. (1990). The management of equity joint ventures in China. Beijing-EC Management Institute.

Consejo Privado de Competitividad (20162017). Ciencia, Tecnología e Innovación. Informe Nacional de Competitividad. Bogotá. Recuperado de: http://compite. com.co/informe/informe-nacional-decompetitividad-2016-2017/cienciatecnologia-e-innovacion/

Cotec (2005). Informe COTEC 2005: Tecnología e innovación en España. Madrid: Fundación COTEC para la Innovación Tecnológica.

Damanpour, F. (1991). Organizational Innovation: A meta-analysis of effects of determinants and moderators. Academy of Management Journal, 34, 555 - 590.

Deninson, D. R. (1991). Cultura corporativa. Bogotá: Legis.

Detert, J. R., Schroeder, R. G., \& Muriel, J. J. (2000). A framework for linking culture and improvement initiatives in organizations. Academy of Management Review, 25(4), 850863.

Empresas Públicas de Medellín, EPM, Gerencia Cultura Organizacional (2015). Informe final cultura organizacional en EPM. Medellín: EPM.

Escorsa, P. (2008). De la vigilancia tecnológica a la inteligencia competitiva de las empresas. Barcelona: Universitat Oberta de Catalunya, UOC, conferencia inaugural de los Estudios de Información y Documentación de la UOC del segundo semestre del curso 2001-2002 (15 de septiembre de 2001). Recuperado de: http:// www.uoc.edu/web/esp/art/uoc/escorsa0202/ escorsa0202_imp.html

Escorsa, P., \& Valls, J. (2001). Tecnología e innovación en la empresa. Barcelona: Ediciones UPC.

Gurteen, D. (1998). Knowledge, creativity and innovation. Journal of Knowledge Management, 2(1), 5-13. Doi: $10.1108 / 13673279810800744$

Hartmann, A. (2006). The role of organizational culture in motivating innovative behaviour in construction firms. Construction innovation, 6(3), 159-172. Doi: $\underline{10.1108 / 14714170610710712}$

Hofstede, G., Hofstede, G. H., \& Minkov, M. (1999). Culturas y organizaciones. El software mental. Madrid: Alianza.

Jakobiak, F. (1992). Exemples commentés de veille technologique. Les Editions d'Organisation, Paris.

Jamrog, J., Vickers, M., \& Bear, D. (2006). Building and sustaining a culture that supports innovation. Human Resource Planning, 29(3), 9-19.

Kimberly, J. R., \& Evanisko, M. J. (1981). Organizational innovation: The influence of individual, organizational, and contextual factors on hospital adoption of technological and administrative innovations. Academy of management journal, 24(4), 689-713.

Lemon, M., \& Sahota, P. S. (2004). Organizational culture as a knowledge repository for increased innovative capacity. Technovation, 24(6), 483498. Doi: 10.1016/S0166-4972(02)00102-5

Lesca, H. (1994) Veille stratégique, l'intelligence de l'entreprise. Aster, Gières.

López, C., Díaz Fúnez, P. A., \& Robledo Velásquez, J. (2016). La organización informal y sus efectos en las capacidades de innovación. Universidad \& Empresa, 17(28), 191-217. Doi: 10.12804/ rev.univ.empresa.28.2015.09.

López, C. (2014). Factores psicosociales que 
caracterizan a los equipos innovadores: un modelo multinivel de evaluación. Revista Interamericana de Psicología Ocupacional, 33(1), $11-30$.

Madri+d, CEIM y Comunidad de Madrid (1995). La innovación: un factor clave para la competitividad, ¿Factor de competitividad? Madrid: Madri+d, CEIM y Comunidad de Madrid. Recuperado de: www.oei.es/historico/ salactsi/libro9.pdf

Martinet, B., \& Marti, Y-M. (1995). L'intelligence économique. Les yeux et les oreilles de l'entreprise. Les Editions d'Organisation, Paris.

Martins, E. C., \& Terblanche, F. (2003). Building organisational culture that stimulates creativity and innovation. European Journal of Innovation Management, 6(1), 64-74. Doi: $10.1108 / 14601060310456337$

McLean, L. D. (2005). Organizational culture's influence on creativity and innovation: a review of the literature and implications for human resource development. Advances in Developing Human Resources, 7(2), 226-246.

Morcillo, P. (2007). Cultura e innovación empresarial: la conexión perfecta. Madrid: Thomson.

Mumford, M. (2000). Managing creative people: Strategies and tactics for innovation. Human Resource Management Review, 10 (3), 313 351.

Naranjo-Valencia, J. C., \& Calderón Hernández, G. (2015). Cultura de la innovación: una propuesta de transformación cultural. Estudios Gerenciales, 31(135), 1-14. Doi : 10.1016/j. estger.2014.12.005

Naranjo-Valencia, J. C., Jiménez-Jiménez, D., \& SanzValle, R. (2011). Innovation or imitation? The role of organizational culture. Management Decision, 48(1), 55-72.

Organización para la Cooperación y el Desarrollo Económicos, OCDE (2005). Manual de Oslo. Guía para la recogida e interpretación de datos sobre innovación $3^{\mathrm{a}}$ ed. Madrid: OCDE y Grupo TRAGSA. Recuperado de: http://www.conacyt.gov.py/sites/default/files/ detiec/concurso/Manual_de_Oslo.pdf

Ponti, F. (2009). Los siete movimientos de la innovación. Bogotá: Norma.
Rothwell, R. (1994). Towards the fifth-generation innovation process. International marketing review, 11(1), 7-31.

Rouach, D. (1996) La veille technologique et l'intelligence économique. PUF Que sais-je? Paris.

Saleh, S. D., \& Wang, C. K. (1993). The management of innovation: strategy, structure and organizational climate. IEEE Transactions on Engineering Management, 40(1), 14-21.

Schatsky, D., \& Schwartz, J. (2015). Tendencias globales de capital humano. Liderando en el nuevo mundo del trabajo. Oakland, CA: Deloitte University Press. Recuperado de: https://www2.deloitte.com/content/dam/ Deloitte/ar/Documents/human-capital/ Tendencias $\% 20$ Globales $\% 20$ en $\% 20$ Capital\%20Humano\%202015.pdf

Schein, E. H. (1988). La cultura empresarial y el liderazgo: una visión dinámica. Barcelona: Plaza y Janés.

Schein, E. H. (1992). Organizational culture and ledership, $2^{\mathrm{a}}$ ed. San Francisco: Jossey-Bass.

Schneider, B., Gunnarson, S. K., \& Niles-Jolly, K. (1994). Creating the climate and culture of success. Organizational Dynamics, 23(1), 17-29. Doi: http://dx.doi.org/10.1016/00902616(94)90085-X

Shrivastava, P., \& Souder, W. (1987). The strategic management of technological innovations: A review and a model. Journal of Management Studies, 24(1), 25-41.

Shumpeter, J. A. (1942). Capitalismo, socialismo y democracia. Madrid: Folio.

Twiss, B. C. (1986) Conference Reports. R\&D Management. Doi: 10.1111/j.1467-9310.1986. th01180-X

Unsworth, K. L. (2001). Unpacking creativity. Academy of Management Review, 26(2), 286297. Doi: 10.5465/AMR.2001.43780

Unsworth, K. L., Brown, H., \& McGuire, L. (2000). Employee innovation: the roles of idea generation and idea implementation. En SIOP Conference 2000, New Orleans, Louisiana, april 14-16.

Unsworth, K. L., \& Parker, S. K. (2003). Proactivity and innovation: promoting a new workforce 
for the new workplace. En D. Holman, T.D. Wall, C. Clegg, P. Sparrow, \& A. Howard (Eds), The new workplace: people, technology and organization. A handbook and guide to the human impact of modern working practices (pp.175-196). Chichester: John Wiley \& Sons.

Unsworth, K. L., Wall, T. D., \& Carter, A. J. (2005). Creativity requirement: a neglected construct in the field of employee creativity. Group \& Organization Management, 30(5), 541-560.

Unsworth, K. L., \& Clegg, C. W. (2010). Why do employees undertake creative action? Journal of Occupational \& Organizational Psychology, 83(1), 77-99.

Velasco, Zamanillo, \& Gurutze (2007). Evolución de los modelos sobre el proceso de innovación: Desde el modelo lineal hasta los sistemas de innovación. XX Congreso anual de AEDEM, Vol. 2, 2007. Disponible en: https://dialnet. unirioja.es/descarga/articulo/2499438.pdf

Wallach, E. (1983). Individuals and organizations: the cultural match. Training and Development Journal, 37(2), 29-36. 